\title{
Luck Hard
}

\section{Les Wicks}

My doctor has warned

I must face an occasional

illogical exuberance, the piano in numbers.

It should be stridently ignored -

an anticipated side effect

of "medical care".

Ignore the Bad Thoughts

during a commercial break.

4 is a sharp number, we are

impaled impalas.

Always a fine line, suppression of mind (the

filthy brumby) and a need to be open, queerly qwerty.

Strange needs to be cultivated 
and suppressed with equal vigour.

An interested mind is a nuclear reactor.

Hands upon the wheel

this seal has clapped for fish. Keratosis Moses.

The ultrasound technician noted fat around the pancreas common for men of my age.

The spleen sits high in the viscera -

a statistical variant in

$20-30 \%$ of people as was the range of difference

in liver lobes. Prostate fine.

Many of our patients contracted this 25 or so years ago

but this gentleman has looked after himself and it shows.

Extraordinary to be dissected like this

before my first coffee. There is nothing fast about fasting.

Hungry numerals.

So much luck! Imagine

the starling... all possible names and someone chose

star-ling for this little pest its coat of remote luminance, planetarium across the ratty spine.

None such for me, a flour bag name old

Robert Wickes stole sheep both there and here/

convict mainstream 1789, crosshatch back lost

even an e in his penitence. 
He married and grew apples across much of North Ryde. Lucky Rob, lucky Sarah. Land Ho!

Children of rich folk

by a statistically sharp (oh but silky) formula are likely to be rich folk.

My daughter joined 7\% of us who voted Green just

like her folks she got a degree and wastes her time on art, sitting in the sun on this propitious afternoon.

These are the best days

our genetics (a nutty mix of digits)

the stockmarket

surf, the coffee.

I'm being irrational

even $\pi$ gets exhausted,

but that's not the point.

Shipwrecked on dimples -

Hippie Tom, Commander Amanda, Artgal Rubberlines and Pastor Blast.

Leak and repeat, the orchestral cacophony. I won't grope for an ending (you can't count the growth rings before the cut). We'll take it easy under the canopy of relief. 